\title{
Intoxication caused by iodoform gauze packing in necrotizing fasciitis
}

\author{
Kensuke Nakamura, Ryota Inokuchi, Hidenobu Fujita, Takahiro Hiruma, Takehiro Matsubara, \\ Yukio Tanaka, Naoki Yahagi
}

\begin{abstract}
While iodoform gauze has a good antiseptic effect, cases of iodoform toxicity are not common. Iodoform can cause neurological symptoms such as disturbance of consciousness, and intoxication occurs with relative ease under certain suitable conditions. Medical practitioners may often misdiagnose disturbance of consciousness for various reasons, and the actual cause of disturbance of consciousness should be clarified. We now report a case of prolonged disturbance of consciousness caused by the use of iodoform gauze in the treatment of necrotizing fasciitis. A 70-year-old diabetic female, who used insulin injections, acquired necrotizing fasciitis in her left buttock and underwent surgery involving release of purulent discharge and debridement. The surgical area was packed with iodoform gauze following surgery, and the gauze was replaced daily. The patient's general condition gradually improved, but disturbance of consciousness, around Glasgow coma scale E2VTM4, was prolonged. We discontinued iodoform gauze treatment, following which consciousness improved slowly. The blood serum iodine level was too high. Although we often use iodoform gauze as an effective treatment for infected sites, we must take heed of its potential toxic neurological effects.
\end{abstract}

Key words: (1) iodoform gauze, (2) intoxication, (3) necrotizing fasciitis

\section{Introduction}

Iodoform gauze is an efficient tool in wound care with considerable antiseptic effect. In wounds, iodoform is broken down to iodine, which is the active agent in antisepsis ${ }^{1}$. Iodoform is especially effective for infectious wounds, such as dirty or purulent sites, and its antimicrobial activity has been evaluated ${ }^{1)}$. However, its toxic effects, chiefly neurological, are relatively unknown. We report a case of necrotizing fasciitis treated with iodoform gauze and presenting with persistent disturbance of consciousness. The initial differential diagnosis of disturbance of consciousness is difficult. All medical practitioners using iodoform gauze should be fully aware of its potential toxic effects.

\section{Case}

A 70 -year-old female presented with fever $\left(40.0^{\circ} \mathrm{C}\right)$, general fatigue for a 72-hr duration and lumbar pain. Her past medical history included hypertension, hyperlipidaemia, chronic hepatitis $\mathrm{C}$ and diabetes for a period of 30 years. She had been using injectable insulin for 5 years, and had acquired diabetic nephropathy stage IIIA. She was admitted to the Department of Internal Medicine in our hospital. She was administered cefotaxime because urinary tract infection was suspected, but her condition did not improve. Two days after admission, she complained of pain in her left buttock, which became red and swollen. Fluctuation or crepitation was not observed. CT scan showed soft tissue inflammation with free air in the left buttock (Fig. 1). We made a diagnosis of necrotizing fasciitis, and surgically opened the area, released the pus and performed debridement under general anaesthesia. Her anus and rectum were intact. The patient was immediately transferred to the ICU when she was still under intubation, and then her wound was packed with iodoform gauze, which was changed at least once daily. The area was washed regularly to remove faecal contamination, and Flexi-Seal ${ }^{\circledR}$ was applied to prevent faecal contamination less.

Fig. 2 shows the clinical course after the surgery. Although catecholamine was initially administered to counteract septic shock, the patient's general condition gradually improved and catecholamine was discontinued on the 14th postoperative day. Purulence gradually reduced, and the area became a clean open wound. As it became evident that long-term intubation and mechanical ventilation would be required, tracheotomy was 
performed on the 10th postoperative day, and sedative medication was withdrawn. Respiratory function improved, and artificial ventilation ceased on the 16th postoperative day. However, disturbance of consciousness, around Japan coma scale II-30, Glasgow coma scale E2VTM4, became prolonged following discontinuation of sedation. The results of brain CT, MRI, and cerebrospinal fluid examination were within the normal range, and electroencephalography showed a generalised slow wave. Although differential diagnosis was difficult, iodoform gauze toxicity was suspected, and iodoform gauze treatment was discontinued on the 26th postoperative day. On the same day, the blood iodine level was

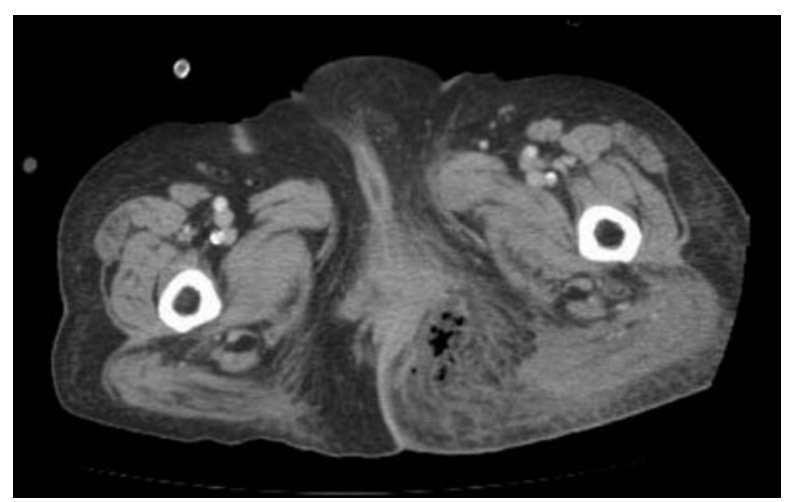

Fig. 1 The enhanced CT

The soft tissue of her left hip was enhanced and got high density, with free air.
$680 \mu \mathrm{g} / \mathrm{d} l$, which is abnormally high. Thyroid function was as follows: thyroid stimulating hormone, TSH, 0.45 $\mu \mathrm{IU} / \mathrm{m} l$ (low); free thyroxine FT4, $3.87 \mathrm{ng} / \mathrm{d} l$ (elevated); and free triiodothyronine FT3, $3.4 \mathrm{pg} / \mathrm{d} l$ (elevated slightly). Consciousness slowly improved following discontinuation of iodoform gauze treatment, and the patient became almost lucid by the 40th postoperative day. The blood iodine level reduced steadily from 680 $\mu \mathrm{g} / \mathrm{d} l$ (26th day) to $88.3 \mu \mathrm{g} / \mathrm{d} l$ (37th day), and then to $6.3 \mu \mathrm{g} / \mathrm{d} l$ (56th day). The wound was treated with sulfadiazine silver cream and dimethyl isopropylazulene ointment after the discontinuation of iodoform gauze treatment.

The patient recovered after rehabilitation, and could walk with assistance. Formation of granulation tissue occurred satisfactorily, with the use of trafermin spray, and the wound was perfectly epithelialised 2 months later.

\section{Discussion}

Iodoform toxicity was first reported in a case presenting with delirium, vomiting, and arrhythmia by Muir et al. in $1903^{2}$ ), and since then, there have been several other reports on the same issue. In Japan, however, approximately only 20 reports regarding iodoform toxicity have been published, indicating that its toxicity is relatively unknown. However, this summary count may be misleading, because potential cases may be misdiagnosed as either ICU delirium or prolonged sedative agents.

The symptom of iodoform toxicity include headache, disorientation, delirium and coma, i.e. central nervous

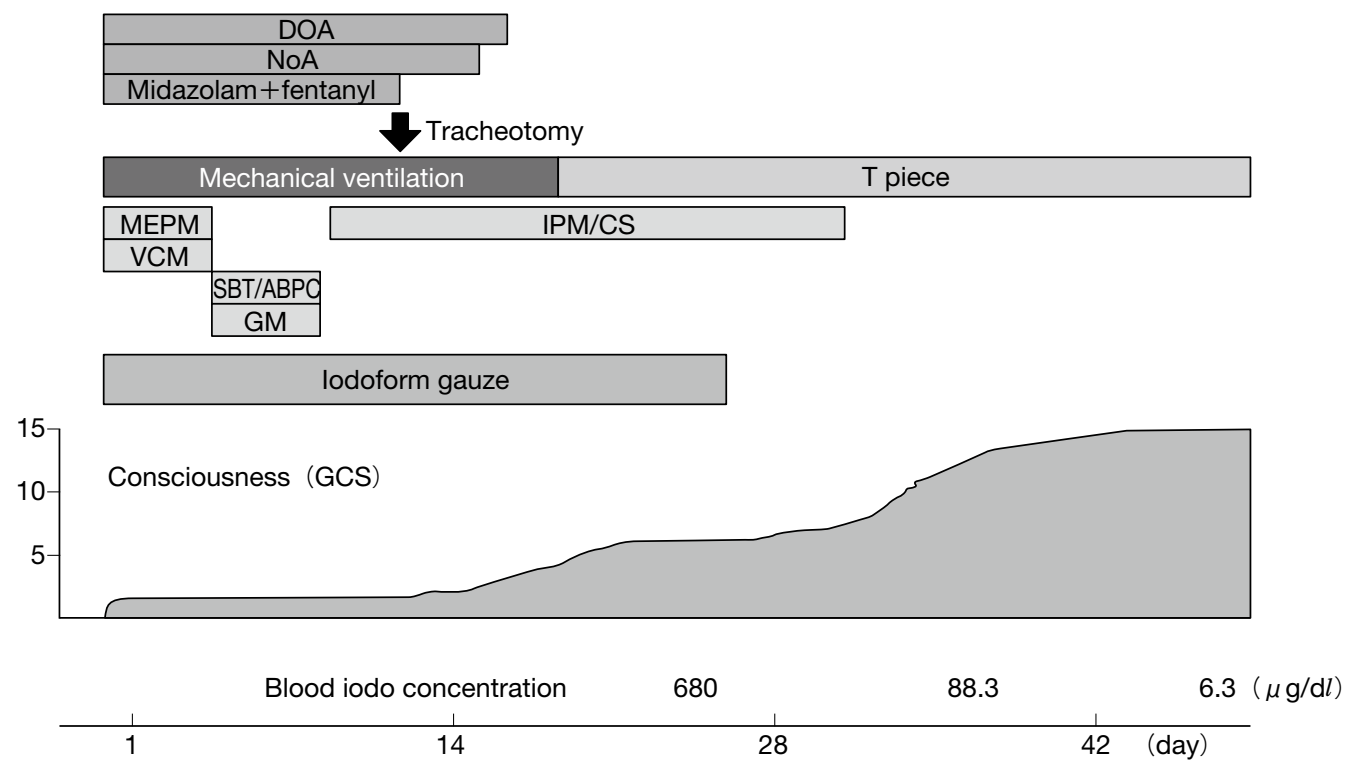

Fig. 2 Clinical course

After surgery, the patient's general condition gradually improved. But disturbance of consciousness was prolonged following discontinuation of sedation. We stopped the iodoform gauze treatment on the 26th day. Then consciousness slowly improved, and the patient became almost lucid by the 40th postoperative day. The blood iodine level reduced from $680 \mu \mathrm{g} / \mathrm{d} l$ (26th day) to $88.3 \mu \mathrm{g} / \mathrm{d} l$ (37th day) and to $6.3 \mu \mathrm{g} / \mathrm{d} l$ (56th day). DOA, dopamine; GCS, Glasgow coma scale; GM, gentamicin; IPM/CS, imipenem / cilastatin; MEPM, meropenem; NoA, noradrenaline; SBT/ABPC, sulbactam/ampicillin; VCM, vancomycin. 
system manifestations, most of which are reversible. Previous reports suggested that recovery time from discontinuation of iodoform treatment was 2 weeks to 1 month, which is a finding corroborated in this case. Although abnormality of thyroid function can occur, overactive and underactive cases have both been reported.

The mechanism of toxicity caused by the use of iodoform is not due to the direct effect of iodine, but it is considered that iodoform $\left(\mathrm{CHI}_{3}\right)$ is similar to chloroform $\left(\mathrm{CHCl}_{3}\right)$ in molecular structure and has a similar anaesthetic effect. Iodoform and diiodomethane, a metabolic product of iodoform, are lipid soluble and may easily cause central nervous system manifestations by crossing the blood-brain barrier ${ }^{3}$. Bearing out the fact that any direct effect of iodine was not involved in this case, the distinctive symptoms of iodine toxicity include disturbance of consciousness, diarrhoea, liver dysfunction, renal failure, and metabolic acidosis, most of which are not the symptoms associated with iodoform toxicity. The blood iodine level in iodine toxicity is 10 times higher than that in iodoform.

As the blood iodoform level can not be measured directly ${ }^{4)}$, iodoform toxicity is diagnosed based on the blood iodine level. The blood iodine level in iodoform toxicity has been reported to be between $31.6-977 \mu \mathrm{g} / \mathrm{d} l$ (normal range: $4-9 \mu \mathrm{g} / \mathrm{d} l$ ), with one case being reported as having a level as high as $6,280 \mu \mathrm{g} / \mathrm{d} l^{5}$. The blood iodine level in the patient in our case was high enough to cause toxicity.

Patients may be prone to toxicity when the daily use of iodoform produces $>2 \mathrm{~g}$ of iodine ${ }^{6}$. . In the present case, each application of iodoform gauze generated approximately $3 \mathrm{~g}$ of iodine, since the wound area was relatively large. Because the wound was close to the patient's anus, the gauze was replaced every time when fecal contamination occurred. Thus, the number of used gauze was greater than usual. In total, we estimate that $80-100 \mathrm{~g}$ of iodine was released into the wound. Although iodoform gauze is one of the most effective treatments for necrotizing fasciitis, gas gangrerne, and Fournier's syndrome, we must be mindful of the potential dangers of iodoform toxicity when repeated dressing is mandated to prevent faecal contamination.

Furthermore, renal dysfunction is a significant risk factor in iodoform toxicity, since iodoform is metabolised by the kidney. Instructions accompanying the commercial iodoform preparation list also indicate patients with renal dysfunction as a contraindication for use. The patient in our case had acquired renal dysfunction through diabetic nephropathy, which worsened through sepsis, and therefore blood iodoform levels may have been higher than otherwise expected. According to a past report ${ }^{7}$, several patients with diabetes or renal dysfunction have developed iodoform toxicity, since the primary disease was often gas gangrene. Medical practitioners must be especially careful when iodoform is used in patients with renal dysfunction. Although iodoform toxicity is reversible, haemodialysis is an effective treatment for its toxicity because of its beneficial effects on renal metabolism ${ }^{8)}$.

\section{Conclusion}

Iodoform gauze is one of the effective treatments for the infected wounds. However, its intoxication is occurred with relative ease and often misdiagnosed or overlooked, when medical practitioners know little of it. We should know the manifestation and conditions in which the symptoms develop easily.

\section{Acknowledgements}

The content of this article was presented at the 35 th Annual Meeting of the Japanese Society of Intensive Care Medicine (Tokyo, 2008).

\section{References}

1) Sakagami Y, Shimura H, Yokoyama H, et al. The evaluation of antibacterial activity with iodoform gauze. Proceedings of Osaka Prefectural Institute of Public Health. Education of pharmaceutical affairs 1988;22:31-4.

2) Muir AH, Glasg CB. A case of iodoform poisoning. Lancet 1903;1:960.

3) Roy PM, Harry P, Cailleux A, et al. Dangers of bismuth iodoform paraffin paste. Lancet 1994;344:1708.

4) Sharma RR, Cast IP, Redferm RM, et al. Extradural application of bismuth iodoform paraffin paste causing relapsing bismuth encephalopathy: a case report with CT and MRI studies. J Neurol Neurosurg Psychiatry 1994;57:990-3.

5) Araki K, Hirakawa N, Kosugi T, et al. Iodoform intoxication ; a case report of prolonged consciousness disturbance in a patient with a high plasma iodine level. Fukuoka Igaku Zassi 2007; 98:397-401.

6) Osal A, Pratt R. The United States dispensatory, 27th Edition. Philadelphia: JB Lippincott Co; 1973. p. 629-30.

7) Numata S, Murayama Y, Makino M, et al. Temporary stupor in a patient treated with iodoform gauze for mediastinitis after coronary artery bypass grafting. Interact Cardiovasc Thorac Surg 2004;3:309-10.

8) Kanakiriya S, De Chazal I, Nath KA, et al. Iodine toxicity treated with hemodialysis and continuous venovenous hemodiafiltration. Am J Kidney Dis 2003;41:702-8. 